\title{
Circulant and Skew-Circulant Matrices as New Normal-Form Realization of IIR Digital Filters
}

\author{
VINCENT C. LIU, STUDENT MEMBER, IEEE, AND P. P. VAIDYANATHAN, SENIOR MEMBER, IEEE
}

\begin{abstract}
Normal-form fixed-point state-space realizations of IIR filters are known to be free from both overflow oscillations and roundoff limit cycles, provided magnitude truncation type of arithmetic is used together with two's complement overflow features. The eigenvalues of the state transition matrix have low sensitivity. In this paper two new normal-form realizations are presented which utilize circulant and skew-circulant matrices as their state transition matrices. The advantage of these realizations is that the $\boldsymbol{A}$-matrix has only $N$ (rather than $N^{2}$ ) distinct elements, and is amenable to efficient memory-oriented implementation. The problem of scaling the internal signals in these structures is addressed and an approximate solution can be obtained through a numerical optimization method. Several numerical examples are included.
\end{abstract}

\section{INTRODUCTION}

I $\mathrm{N}$ THE fixed-point implementation of IIR filters, several undesirable effects occur due to the finite wordlength of the implementation. State-space approach has been used in the past to minimize such effects. In particular, normal-form digital filters have been shown to possess several good qualities with regard to finite wordlength effects such as the absence of limit cycles, and low coefficient sensitivity [1], [2], [7]. The condition for the absence of overflow oscillations had been derived by Barnes and Fam [1], which is to restrict the $l_{2}$ norm of the state transition matrix to be less than unity, and normal-form structures satisfy this requirement. It had been pointed out further by Jackson [6] that the same condition would also lead to the suppression of quantization limit cycles when the quantization is done by magnitude truncation. So with the use of magnitude truncation type of quantization arithmetic together with two's complement overflow features, both types of limit cycles may be eliminated from normalform filters. Most of the normal-form structures that have been presented in the past made use of second order normal sections as building blocks. They consist of either cascaded or parallel connections of second-order sections $[1],[3]$.

Manuscript received August 29, 1985; revised May 5, 1986 and July 22 1987. This work was supported in part by the National Science Foundation under Grant ECS 84-04245 and in part by CalTech's programs in advanced technology sponsored by Aerojet General, General Motors, GTE, and TRW. V. C. Liu was under a Schlumberger Fellowship for the period during which this work was performed. This paper was recommended by Associate Editor A. N. Venetsanopoulos.

The authors are with the Department of Electrical Engineering, California Institute of Technology, Pasadena, CA 91125.

IEEE Log Number 8820490 .
In this paper, we shall present a new family of normalform state-space structures. The method used allows us to synthesize in normal form, most IIR transfer functions. We shall refer to the structures in this paper as circulant and skew-circulant forms, since the state transition matrices involved are either circulant or skew-circulant matrices. The well known second-order coupled form [4] is a special case of the skew-circulant form.

In Section II, the low sensitivity and limit-cycle free property of normal-form structures in general are reviewed. In addition we introduce a new measure for the eigenvalue sensitivity of a state transition matrix. In Section III, the method by which circulant and skew-circulant forms are derived is presented. We then address the problem of scaling these structures in Section IV, which also includes a study of roundoff. noise in these filters.

Although the circulant and skew-circulant realizations have in general $N^{2}$ nonzero entries in the state transition matrix, yet there are only $N$ distinct elements and the circulant structure of the matrix makes these filters amenable to efficient memory-oriented hardware implementations. It has been recognized in the past that circular convolution is a key operation in a number of signal processing algorithms, including finite and infinite length linear convolutions [10], [17]. The use of circular convolution in recursive linear filtering is particularly noticeable in block realization of IIR filters [18]-[20]. If we have a signal processing hardware which primarily implements circular convolutions, then it offers a wide range of applications. The result of this paper adds one more application to this list, namely, a limit-cycle free, low eigenvalue sensitivity implementation of IIR filters. In this connection, it is worth noting that block realization of digital filters in the state-space context has been studied by Barnes et al. [21]; it is easily verified that the "block version" of a circulant realization retains the circulant nature, since the state transition matrix $\boldsymbol{A}$ is merely replaced by $A^{L}$ where $L$ is the block length.

Purely from the viewpoint of computational complexity, (i.e., the number of multiplications and additions per sample), the structures introduced here are not necessarily more efficient than the second-order block normal form in [1]. However, as mentioned above, there are at least two contexts where the results of this paper can be useful: 
memory oriented implementations, and implementations based on convolution building blocks.

\section{Review and a New Eigenvalue SENSITIVITY MEASURE}

A state-space structure for an IIR filter with input $u(n)$ and output $y(n)$ is characterized by the equations [5]

$$
\begin{gathered}
\boldsymbol{x}(n+1)=\boldsymbol{A} \boldsymbol{x}(n)+\boldsymbol{B} u(n) \\
y(n)=\boldsymbol{C} \boldsymbol{x}(n)+d u(n) .
\end{gathered}
$$

In this paper, we shall deal with single-input-single-output systems where $\boldsymbol{A}=\left[a_{k l}\right]$ is an $N \times N$ matrix with $0 \leqslant$ $k, l \leqslant N-1 . \quad B$ is $N \times 1$, and $C$ is $1 \times N$. Let $\lambda_{i}$ be an eigenvalue of $\boldsymbol{A}$. We shall denote the column eigenvector associated with $\lambda_{i}$ as $\Phi_{i}$, while ${ }^{1} \Psi_{i}^{\dagger}$ will denote the corresponding row eigenvector, i.e.,

$$
A \Phi_{i}=\lambda_{i} \Phi_{i} \text { and } \Psi_{i}^{\dagger} A=\lambda_{i} \Psi_{i}^{\dagger} .
$$

Throughout this paper we shall assume that $\Psi_{i}$ and $\Phi_{i}$ are scaled such that $\Psi_{i}^{\dagger} \Phi_{i}=1$. The $k$ th components of $\Phi_{i}$ and $\Psi_{i}$ are denoted by $\phi_{i}(k)$ and $\psi_{i}(k)$, respectively. The transfer function $H(z)$ of the IIR filter is related to the state-space parameters by

$$
H(z)=d+C(z \mathbf{I}-\boldsymbol{A})^{-1} \boldsymbol{B} .
$$

It is well known that the following similarity transformation:

$$
\boldsymbol{A}^{\prime}=\boldsymbol{T A} \boldsymbol{T}^{-1} \quad \boldsymbol{B}^{\prime}=\boldsymbol{T B} \quad \boldsymbol{C}^{\prime}=\boldsymbol{C} \boldsymbol{T}^{-1}
$$

leaves $H(z)$ unchanged. The eigenvalues of $\boldsymbol{A}$ correspond to the poles of the transfer function $H(z)$. A normal form realization is one in which the state transition matrix $\boldsymbol{A}$ is a normal matrix. We shall assume that the poles $H(z)$ are all distinct, because a minimal system with non-distinct poles does not have a normal-form realization. This can be seen from the fact that if the $N \times N$ state transition matrix is diagonalizable and has less than $N$ distinct eigenvalues, then such a realization cannot be minimal. Since a normal matrix is always diagonalizable [8], any normal-form realization having less than $N$ distinct eigenvalues is necessarily non-minimal.

In state-space implementation, it is possible to avoid both overflow oscillations and quantization limit cycles by requiring an upper bound of unity on the norm of the state transition matrix $\boldsymbol{A}$ [1]. Barnes and Fam have introduced the novel minimum-norm structures [1] by constraining $\boldsymbol{A}$ to be a normal matrix. Mills, Mullis, and Roberts [7] have obtained a general set of sufficient conditions in terms of $\boldsymbol{A}$, in order to suppress overflow oscillations. As pointed out by Jackson [6], the same conditions would eliminate quantization limit cycles when magnitude truncation is used. The conditions required in [7] have been improved in

\footnotetext{
${ }^{1}$ Superscript $T$ denotes transposition and superscript $\dagger$ denotes transposed conjugation.
}

[16] and can be used to explain the absence of limit cycles in several well-known structures, such as the wave-digital filters, orthogonal filters, cascaded lattice structures, normal-form structures and second-order minimum-noise structures. If the quantization scheme is of the magnitude truncation type, along with 2's complement overflow characteristics, then both quantization limit cycles and overflow oscillations can be suppressed if the $A$ matrix satisfies the conditions given in [16]. Such an arithmetic scheme involves the use of two's complement representation for negative numbers, and the quantization is done in two different ways depending on the sign of the number to be quantized. If the number is positive, a straightforward magnitude truncation is performed; if the number is negative, then it is also truncated, but at the end $2^{-b}$ is added to the truncated number $b$ being the wordlength of the implementation.

The norm of $\boldsymbol{A}$, denoted by $\|\boldsymbol{A}\|_{2}$, is defined to be

$$
\|\boldsymbol{A}\|_{2}^{2}=\max _{x \neq 0} \frac{\boldsymbol{x}^{\dagger} \boldsymbol{A}^{\dagger} \boldsymbol{A} \boldsymbol{x}}{\boldsymbol{x}^{\dagger} \boldsymbol{x}} .
$$

The norm $\|A\|_{2}$ is at least as large as the magnitude of the dominant eigenvalue (i.e., the spectral radius) of $\boldsymbol{A}$ :

$$
\|\boldsymbol{A}\|_{2} \geqslant \max |\lambda(\boldsymbol{A})| \text {. }
$$

A normal matrix [8] has its norm strictly equal to its spectral radius, therefore, $\|\boldsymbol{A}\|_{2}<1$ as long as the eigenvalues of $\boldsymbol{A}$ are inside the unit circle. So provided the filter is stable, a normal-form realization will always satisfy the conditions given in [7], [16]. Hence it is possible to suppress limit cycles in these structures. The purpose of this paper is to introduce new normal-form filters and to explore their advantages.

A normal matrix is any square matrix that satisfies the condition $\boldsymbol{A}^{\dagger} \boldsymbol{A}=\boldsymbol{A} \boldsymbol{A}^{\dagger}$. This condition in turn holds if and only if $\boldsymbol{A}$ has a complete set of $N$ orthogonal eigenvectors [8]. This is the same as saying $\boldsymbol{A}$ is diagonalizable by a unitary matrix. If $\boldsymbol{A}$ is normal, $\left\{\Phi_{i}\right\}$ is a complete set of orthogonal eigenvectors. Then it can be shown that $\Phi_{i}=\Psi_{i}$ for each $i$. Conversely, if for each $i \Phi_{i}=\Psi_{i}$ within a scalar factor, then $\boldsymbol{A}$ is necessarily normal. Typical examples of normal matrices are Hermitian matrices, and unitary matrices [8].

In the study of eigenvalue sensitivity, a commonly used global sensitivity measure [2] is defined to be

$$
\sum_{k=0}^{N-1} \sum_{l=0}^{N-1}\left|\frac{\partial \lambda_{i}}{\partial a_{k l}}\right|^{2} .
$$

The above quantity measures the sensitivity of $\lambda_{i}$ with respect to the entries of the matrix $A$. Now, given any matrix $\boldsymbol{A}$ with distinct eigenvalues [2], the eigenvalues always satisfy

$$
\frac{\partial \lambda_{i}}{\partial a_{k l}}=\psi_{i}^{*}(k) \phi_{i}(l) .
$$

$\phi_{i}(k)$ and $\psi_{i}(l)$ are the components of the vectors $\Psi_{i}$ and $\Phi_{i}$, respectively. Using (8), the sensitivity measure in (7) 
becomes [2]

$$
\begin{aligned}
\sum_{k=0}^{N-1} \sum_{l=0}^{N-1}\left|\frac{\partial \lambda_{i}}{\partial a_{k l}}\right|^{2} & =\sum_{k=0}^{N-1} \sum_{l=0}^{N-1}\left|\psi_{i}^{*}(k) \times \phi_{i}(l)\right|^{2} \\
& =\left\|\Psi_{i}\right\|^{2}\left\|\Phi_{i}\right\|^{2} \\
& \geqslant\left(\Psi_{i}^{\dagger} \Phi_{i}\right)^{2} \\
& =1 .
\end{aligned}
$$

Clearly, the lower bound in (9) is achieved if and only if $\Psi_{i}=\Phi_{i}$ upto a scalar factor. Furthermore, in order to achieve the lower bound for all $\lambda_{i}(i=1, \cdots, N)$, it is necessary and sufficient that $\boldsymbol{A}$ should be normal.

\section{A New Measure for Eigenvalue Sensitivity}

We wish to introduce a different measure for the sensitivity of the eigenvalue $\lambda_{i}$. This measure is directly related to the maximum possible amount of deviation an eigenvalue might undergo, when the entries of the matrix are quantized. Let $\lambda_{i}$ be the original eigenvalue and $\lambda_{i}+\Delta \lambda_{i}$ be the eigenvalue after quantization, then to first-order approximation

$$
\Delta \lambda_{i}=\sum_{k=0}^{N-1} \sum_{l=0}^{N-1}\left(\Delta a_{k, l}\right) \frac{\partial \lambda_{i}}{\partial a_{k, l}}
$$

where $\Delta a_{k l}$ represents the quantization error in the $k$ th row, $l$ th column entry of the $\boldsymbol{A}$ matrix. Let $\Delta_{\max }$ be the maximum possible quantization error in the entries of $\boldsymbol{A}$, then

$$
\left|\Delta \lambda_{i}\right| \leqslant \Delta \max \sum_{k=0}^{N-1} \sum_{l=0}^{N-1}\left|\frac{\partial \lambda_{i}}{\partial a_{k, l}}\right|
$$

$\Delta_{\max }$ depends on the quantization scheme chosen, while the summation term is dependent only upon the matrix $\boldsymbol{A}$. We shall define a sensitivity measure for $\lambda_{i}$ with respect to $A$ as

$$
e_{i}(\boldsymbol{A})=\sum_{k=0}^{N-1} \sum_{l=0}^{N-1}\left|\frac{\partial \lambda_{i}}{\partial a_{k, l}}\right|=\sum_{k=0}^{N-1} \sum_{l=0}^{N-1}\left|\psi_{i}(k) \times \phi_{i}(l)\right| .
$$

One may derive several facts about the quantity $e_{i}(A)$ :

1) If $\Psi_{i}$ is scaled by a nonzero factor $\alpha$, and $\Phi_{i}$ scaled by $1 / \alpha$, then $e_{i}(\boldsymbol{A})$ is invariant under such scaling, and the constraint $\Psi_{i}^{\dagger} \Phi_{i}=1$ still holds.

2) For any matrix, $e_{i}(A) \geqslant 1$.

To prove this, suppose for a certain matrix $\boldsymbol{A}$, the quantity $e_{i}(A)$ is less than unity for some $i$. This means

$$
\left(\sum_{k=0}^{N-1}\left|\psi_{i}(k)\right|\right) \times\left(\sum_{l=0}^{N-1}\left|\phi_{i}(l)\right|\right)<1 .
$$

Since $\Psi_{i}$ can be scaled up by a nonzero factor $\alpha$, and $\Phi_{i}$ by $1 / \alpha$ without affecting the value of $e_{i}(A)$, therefore, we can choose $\alpha$ so that $\sum_{k}\left|\psi_{i}(k)\right|=1$; then we must have $\sum_{l}\left|\phi_{i}(l)\right|<1$. This leads to

$$
\begin{gathered}
\sum_{k}\left|\psi_{i}(k)\right|^{2} \leqslant \sum_{k}\left|\psi_{i}(k)\right|=1 \\
\sum_{l}\left|\phi_{i}(l)\right|^{2}<\sum_{l}\left|\phi_{i}(l)\right|<1
\end{gathered}
$$

and by the Cauchy-Schwarz inequality $\left|\Psi^{\dagger} \Phi\right|^{2} \leqslant$ $\|\Psi\|_{2}^{2}\|\Phi\|_{2}^{2}<1$. But this violates the condition $\Psi^{\dagger} \Phi=1$, so the assumption that $e_{i}(A)<1$ is false.

3) If $\boldsymbol{A}$ is a normal matrix, then $e_{i}(\boldsymbol{A}) \leqslant N$.

Recall that for normal matrices $\Psi_{i}=\Phi_{i}$ for all $i$, and $\left\|\Phi_{i}\right\|=1$ since we assume $\Psi_{i}^{\dagger} \Phi_{i}=1$. Let us define the vectors, $\mathbf{1}=(1,1, \cdots, 1)^{T}$ and $\boldsymbol{v}=\left(\left|\phi_{i}(0)\right|,\left|\phi_{i}(1)\right|, \cdots, \mid \phi_{i}(N\right.$ $-1) \mid)^{T}$, then

$$
e_{i}(\boldsymbol{A})=\left(\sum_{k=0}^{N-1}\left|\phi_{i}(k)\right|\right)^{2}=\left(\mathbf{1}^{T} \boldsymbol{v}\right)^{2} .
$$

By the Cauchy-Schwartz inequality,

$$
\left(\mathbf{1}^{T} \boldsymbol{v}\right)^{2} \leqslant\|\mathbf{1}\|_{2}^{2}\|\boldsymbol{v}\|_{2}^{2}=N\|\boldsymbol{v}\|_{2}^{2}=N\left\|\Phi_{i}\right\|_{2}^{2}=N .
$$

Combining (15) and (16) we conclude that $e_{i}(A) \leqslant N$. This is true for all the eigenvalues of a normal matrix.

Thus a normal matrix is well conditioned in the sense that its sensitivity measure (12) cannot exceed $N$. As examples, the minimum-norm structure in [1] has $e(A)=2$, and circulant matrices have $e(\boldsymbol{A})=N$.

\section{Circulant and Skew-Circulant State-Space Realizations}

A $3 \times 3$ circulant matrix has the following form:

$$
\left(\begin{array}{lll}
a_{0} & a_{1} & a_{2} \\
a_{2} & a_{0} & a_{1} \\
a_{1} & a_{2} & a_{0}
\end{array}\right)
$$

In general, a $N \times N$ matrix $\left[a_{m, n}\right]$ is defined to be circulant if

$$
a_{m, n}= \begin{cases}a_{0, n-m}, & m \leqslant n \\ a_{0, n-m+N}, & m>n .\end{cases}
$$

Notice that a circulant matrix is completely characterized by its zeroth row. Given any $N \times N$ circulant matrix $C$, it can always be diagonalized [14] by the $N \times N$ DFT matrix,

$$
C \mathscr{W}=\mathscr{W} \Lambda
$$

where $\mathscr{W}=\left[W^{k n}\right]$, with $W=e^{-j 2 \pi / N}$. Thus the columns of $\mathscr{W}$ are the eigenvectors of $C$, and $\Lambda$ is the diagonal matrix containing $\left(\lambda_{0}, \lambda_{1}, \cdots \lambda_{N-1}\right)$ which are the eigenvalues of $C$. From (18) it can be seen that the eigenvalues are obtainable by performing a DFT on the top row of the matrix $C$. Conversely, given any diagonal matrix $\Lambda$, we can always form a circulant matrix $C$ as $\mathscr{W} \Lambda \mathscr{W}^{-1}$. In practice $\boldsymbol{C}$ can be found by computing the inverse DFT of the sequence $\left(\lambda_{0}, \lambda_{1}, \cdots, \lambda_{N-1}\right)$ which gives us the top row of $C$. 
Similarly, a matrix is defined to be skew-circulant if

$$
a_{m, n}= \begin{cases}a_{0, n-m}, & m \leqslant n \\ -a_{0, n-m+N}, & m>n .\end{cases}
$$

Both the circulant and skew-circulant matrices are normal and therefore possess all the desirable properties indicated in Section II.

Let $H(z)=P(z) / Q(z)$ be an IIR transfer function. We shall assume that $Q(z)$ has degree $N$, and $P(z)$ has degree $\leqslant N$. Furthermore, let the poles of $H(z)$ be all distinct and denote them by $\lambda_{0}, \lambda_{1} \cdots, \lambda_{N-1}$, then by partial fraction expansion

$$
H(z)=d+\frac{\beta_{0} \gamma_{0}}{z-\lambda_{0}}+\frac{\beta_{1} \gamma_{1}}{z-\lambda_{1}}+\cdots \frac{\beta_{N-1} \gamma_{N-1}}{z-\lambda_{N-1}} .
$$

The product $\beta_{i} \gamma_{i}$ is the residue of $H(z)$ at pole location $\lambda_{i}$. The state-space parameters for this parallel-form structure are

$$
\begin{aligned}
\boldsymbol{A} & =\operatorname{diag}\left[\lambda_{0}, \lambda_{1} \cdots, \lambda_{N-1}\right] \\
\boldsymbol{B} & =\left[\beta_{0}, \beta_{1} \cdots, \beta_{N-1}\right]^{T} \\
\boldsymbol{C} & =\left[\gamma_{0}, \gamma_{1} \cdots, \gamma_{N-1}\right]
\end{aligned}
$$

and $d$ is the constant term in (20). Applying the similarity transformation (4) with $T=\mathscr{W}$, we get

$$
\boldsymbol{A}^{\prime}=\mathscr{W} \boldsymbol{A} \mathscr{W}^{-1} \quad \boldsymbol{B}^{\prime}=\mathscr{W} \boldsymbol{B} \quad C^{\prime}=C \mathscr{W}^{-1} .
$$

The transformed matrix $\boldsymbol{A}^{\prime}$ is circulant, and its top row is simply the inverse DFT of the complex sequence $\left\{\lambda_{0}, \lambda_{1}\right.$, $\left.\cdots, \lambda_{N-1}\right\}$.

$$
a_{0, n}^{\prime}=\frac{1}{N} \sum_{k=0}^{N-1} \lambda_{k} W^{-n k}
$$

classical IIR filters, such as Chebychev, Butterworth, and elliptic filters, have either one real pole in which case the filter order is odd, or no real poles at all in which case the filter has even order.

For a filter with one real pole, a circulant matrix $\boldsymbol{A}^{\prime}$ can be obtained which has real entries only. Starting with the parallel form realization in (21), the complex $\lambda$-sequence can be arranged to have the following symmetry:

$$
\begin{gathered}
\lambda_{0}, \quad \text { real } \\
\lambda_{k}=\lambda_{N-k}^{*}, \quad \text { for } k=1, \cdots, \frac{N-1}{2} .
\end{gathered}
$$

Notice that the order of the filter, $N$, is odd. The inverse DFT of a sequence having the above property will be real. Since the poles are arranged to satisfy (24), their corresponding residues will also be in complex-conjugate pairs. In the parallel form of (21), we have the freedom to choose the $\beta$ 's and $\gamma$ 's arbitrarily, as long as their product equals the corresponding residue of $H(z)$. To generate real matrices $\boldsymbol{B}^{\prime}$ and $\boldsymbol{C}^{\prime}$, it is sufficient to have

$$
\begin{gathered}
\beta_{0}, \quad \text { real } \\
\beta_{k}=\beta_{N-k}^{*}, \quad \text { for } k=1, \cdots, \frac{N-1}{2} .
\end{gathered}
$$

The same symmetry follows for the $\gamma$ 's automatically. Once the values for $\beta$ and $\gamma$ are chosen, $\boldsymbol{B}^{\prime}$ and $\boldsymbol{C}^{\prime}$ are obtained as in (22).

Example 3.1: Consider a fifth-order elliptic low-pass filter with maximum passband attenuation $A_{p}=2 \mathrm{~dB}$, and minimum stopband attenuation $A_{s}=50 \mathrm{~dB}$. The passband cutoff frequency is $0.131 \pi$, while the stopband edge is at $0.185 \pi$. The filter transfer function is given by

$$
H(z)=\frac{2.6019 \times 10^{-3}\left(1-1.30412 z^{-1}+z^{-2}\right)\left(1-1.64983 z^{-1}+z^{-2}\right)\left(1+z^{-1}\right)}{\left(1-1.7906 z^{-1}+0.86809 z^{-2}\right)\left(1-1.80221 z^{-1}+0.96161^{-2}\right)\left(1-0.89734 z^{-1}\right)}
$$

The circulant state-space realization for this filter is shown below:

$$
\begin{aligned}
\boldsymbol{A} & =\left(\begin{array}{ccccc}
0.8980 & -0.1905 & 0.0876 & -0.0854 & 0.1876 \\
0.1876 & 0.8980 & -0.1905 & 0.0876 & -0.0854 \\
\vdots & \vdots & \vdots & \vdots & \vdots \\
-0.1905 & 0.0876 & -0.0854 & 0.1876 & 0.8980
\end{array}\right) \\
\boldsymbol{B} & =\left(\begin{array}{lllll}
0.7028 & -0.2429 & -0.1323 & 0.7081 & 0.5631
\end{array}\right)^{T} \\
\boldsymbol{C} & =\left(\begin{array}{lllll}
0.1406 & 0.1126 & 0.1416 & -0.02646 & -0.04857
\end{array}\right) \\
d & =2.6019 \times 10^{-3} .
\end{aligned}
$$

The remaining rows of $\boldsymbol{A}^{\prime}$ are the circularly shifted versions of this row. If there is no condition imposed on the $\lambda$-sequence, then in general the entries of $\boldsymbol{A}^{\prime}$ are complex numbers.

However, in most situations the coefficients of $H(z)$ are real and consequently the poles of $H(z)$ come either as real numbers or in complex-conjugate pairs. Utilizing this property of IIR functions, we can obtain matrices for (22) which have only real entries. In practice, many of the
For the case of IIR filters having no real poles, the sequence of poles cannot be arranged to have the symmetry as in (24). However it is possible to get

$$
\lambda_{k}=\lambda_{N-k-1}^{*}, \quad \text { for } k=0, \cdots,(N / 2)-1 .
$$

The filter order, $N$, is necessarily even in this case. Let us apply again transformation (22) on the parallel form. The inverse DFT of the $\lambda$-sequence in (27) is complex, and (23) 
becomes

$$
\begin{aligned}
a_{0, n}^{\prime} & =\frac{1}{N} \sum_{k=0}^{(N / 2)-1}\left(\lambda_{k} W^{-k n}+\lambda_{k}^{*} W^{-(N-k-1) n}\right) \\
& =\frac{W^{(n / 2)}}{N} \sum_{k=0}^{(N-2)-1} 2 \operatorname{Re}\left\{\lambda_{k} W^{-\left(k+\frac{1}{2}\right) n}\right\} \\
& =W^{(n / 2)} c(n)
\end{aligned}
$$

where $c(n)$ represents the sum

$$
\frac{1}{N} \sum_{k} 2 \operatorname{Re}\left\{\lambda_{k} W^{-\left(k+\frac{1}{2}\right) n}\right\} .
$$

Since $\boldsymbol{A}^{\prime}$ is circulant, its remaining rows are given by

$$
a_{m, n}^{\prime}= \begin{cases}W^{(n-m) / 2} c(n-m), & \text { if } m \leqslant n \\ W^{(n-m+N) / 2} c(n-m+N), & m>n .\end{cases}
$$

This circulant matrix with complex entries can be converted into a skews-circulant matrix with real entries by applying a diagonal similarity transformation. Thus, define the diagonal matrix $\boldsymbol{D}=\operatorname{diag}\left[1, W^{\frac{1}{2}}, \cdots, W^{\frac{N-1}{2}}\right]$ then $\boldsymbol{A}^{\prime \prime}$ $=\boldsymbol{D} \boldsymbol{A}^{\prime} \boldsymbol{D}^{-1}$ is a skew-circulant matrix, with entries

$$
\begin{aligned}
a_{m, n}^{\prime \prime} & =W^{\frac{m}{2}} a_{m, n}^{\prime} W^{(n / 2)} \\
& = \begin{cases}c(n-m), & \text { if } m \leqslant n \\
-c(n-m+N), & m>n .\end{cases}
\end{aligned}
$$

Example 3.2: The second example is a sixth-order lowpass Butterworth filter, which has been used in [3] for the study of second-order minimum-noise sections. The filter has the following transfer function:

$$
H(z)=\frac{8.5468 \times 10^{-10}\left(1+z^{-1}\right)^{6}}{\left(1-1.96413 z^{-1}+0.96802 z^{-2}\right)\left(1-1.9112 z^{-1}+0.91498 z^{-2}\right)\left(1-1.88191 z^{-1}+0.88563 z^{-2}\right)} .
$$

In this case, $N$ is even and the skew-circulant realization is

$$
\begin{aligned}
\boldsymbol{A} & =\left(\begin{array}{cccccc}
0.95954 & -0.01481 & -0.01086 & -0.01083 & -0.01480 & -0.03855 \\
0.03855 & 0.95954 & -0.01481 & -0.01086 & -0.01083 & -0.01480 \\
\vdots & \vdots & \vdots & \vdots & \vdots & \vdots \\
0.01481 & 0.01086 & 0.01083 & 0.01480 & 0.03855 & 0.95954
\end{array}\right) \\
\boldsymbol{B} & =\left(\begin{array}{lllllll}
0.97076 & -1.07874 & 0.94898 & 0.38069 & 0.016407 & -0.35519
\end{array}\right)^{T} \\
\boldsymbol{C} & =\left(\begin{array}{llllll}
0.16179 & 0.059198 & -0.0027344 & -0.063447 & -0.15816 & 0.17979
\end{array}\right) \\
d & =8.5468 \times 10^{-10} .
\end{aligned}
$$

We wish to point out that for the case of $N=2$, the $2 \times 2$ skew-circulant matrix is exactly the second-order coupled form (or normal form) introduced in [4].

In conclusion, we see that IIR filters having one real pole can be implemented with a circulant state-transition matrix, and IIR filters that contain no real poles will have a skew-circulant state-space implementation. These two cases cover most IIR filters that are commonly used.

Let us now consider IIR filters that have more than one real pole. If $H(z)$ has exactly two real poles, then the order
Now, $\boldsymbol{A}^{\prime}$ will still have the form in (33b), except $D$ will no longer be diagonal, instead it will be block diagonal consisting of second-order circulant blocks. Such a matrix satisfies the normal property, and is an extension of the minimum-norm matrices in [1].

\section{Complexity of Implementation}

State-space structures in general require a large number of multipliers. For example, the matrix-vector product 
$\boldsymbol{A} \boldsymbol{x}(n)$ requires $N^{2}$ multiplications when $\boldsymbol{A}$ is a full matrix. However, with $\boldsymbol{A}$ being circulant, the product $\boldsymbol{A} \boldsymbol{x}(n)$ can be thought of as a circular correlation between $x(n)$ and the first row of $\boldsymbol{A}$. There exists fast algorithms for performing short circular convolutions (and hence correlation) [10], and these can be used to reduce the number of multiplications.

The circular correlation above can also be viewed as the computation of $N$ inner products. One vector in the inner product is always fixed, which is the first row of $\boldsymbol{A}$. And the other vector is $\boldsymbol{x}(n)$ or circularly shifted versions of it. In [11], Peled and Liu have presented an efficient hardware for computing the inner product between a fixed vector and an arbitrary input vector. This is suitable for our purpose here, since the $N$ inner products are all computed with respect to a common vector, namely the first row of $\boldsymbol{A}$. In effect, this means it is only necessary to store the Boolean linear combinations of this common vector in a read-only memory. Only one ROM is needed to compute all $N$ inner products.

\section{Scaling And NoISE ANalysis}

Before analyzing the signal to noise performance of a digital filter structure, we need to scale the structure so that the probability of overflow at each internal node is kept below certain tolerance level. It is sufficient [12] to ensure low overflow probability at nodes that are input to multipliers. In terms of a state-space structure, this means we only need to be concerned with state variable nodes.

Let us define, as in [13], $f_{i}(n)$ to be the impulse response from the filter input to the $i$ th state variable node. A structure is said to be scaled in the $l_{2}$ sense if

$$
\sum_{n=0}^{\infty} f_{i}^{2}(n)=\frac{1}{\delta}, \quad \text { for } i=1, \cdots, N
$$

where $\delta$ is a parameter that relates to the probability of overflow. Define the $\boldsymbol{K}$-matrix as in [13]:

$$
\boldsymbol{K}=\sum_{n=0}^{\infty} \boldsymbol{A}^{n} \boldsymbol{B} \boldsymbol{B}^{T} \boldsymbol{A}^{n^{T}}
$$

Then (35) is equivalent to

$$
[K]_{i, i}=\frac{1}{\delta}, \quad \text { for } i=1, \cdots, N .
$$

State-space structures can be scaled by similarity transformations. When a state space structure $\{\boldsymbol{A}, \boldsymbol{B}, \boldsymbol{C}, d\}$ is transformed into $\left\{\boldsymbol{T A} \boldsymbol{T}^{-1}, \boldsymbol{T B}, \boldsymbol{C} \boldsymbol{T}^{-1}, d\right\}$, the corresponding $\boldsymbol{K}$-matrix is transformed by

$$
\boldsymbol{K}^{\prime}=\boldsymbol{T K} \boldsymbol{T}^{T} .
$$

However, if $\boldsymbol{A}$ is a circulant matrix then the resulting state transition matrix $\boldsymbol{A}^{\prime}$ will not, in general, remain circulant after transformation. If the transformation $\boldsymbol{T}$ itself is a circulant matrix, then due to the commutative property of circulant matrices [14] we get (with $\boldsymbol{A}$ being circulant),

$$
\boldsymbol{A}^{\prime}=\boldsymbol{T A} \boldsymbol{T}^{-1}=\boldsymbol{T} \boldsymbol{T}^{-1} \boldsymbol{A}=\boldsymbol{A} .
$$

Thus the matrix $\boldsymbol{A}$ is unchanged, when the transformation itself is circulant. In order to preserve the circulant nature of $\boldsymbol{A}$, a circulant transformation should be used. But for a given unscaled circulant structure $\{\boldsymbol{A}, \boldsymbol{B}, \boldsymbol{C}\}$, how can we find a transformation that is circulant and such that the transformed matrix $\boldsymbol{T K} \boldsymbol{T}^{T}$ has all diagonal elements satisfying (37)?

Stating the problem in another way, let us denote the first row of $\boldsymbol{T}$ by $\boldsymbol{t}^{T}$, then $\boldsymbol{T}$ can be expressed as

$$
\boldsymbol{T}=\left(\begin{array}{c}
\boldsymbol{t}^{T} \\
\boldsymbol{t}^{T} \boldsymbol{P}^{T} \\
\vdots \\
\boldsymbol{t}^{T}\left(\boldsymbol{P}^{N-1}\right)^{T}
\end{array}\right)
$$

where $\boldsymbol{P}$ is the permutation matrix:

$$
\boldsymbol{P}=\left(\begin{array}{ccccc}
0 & 0 & \cdots & 0 & 1 \\
1 & 0 & \cdots & 0 & 0 \\
\vdots & \vdots & & \vdots & \vdots \\
0 & 0 & \cdots & 1 & 0
\end{array}\right) .
$$

Thus $\boldsymbol{P t}$ is a circularly shifted version of $\boldsymbol{t}$. What is needed is a vector $t$ which will solve the following system of equations:

$$
\begin{aligned}
\boldsymbol{t}^{T} \boldsymbol{K} \boldsymbol{t} & =\frac{1}{\delta} \\
\boldsymbol{t}^{T} \boldsymbol{P}^{T} \boldsymbol{K} \boldsymbol{P} \boldsymbol{t} & =\frac{1}{\delta} \\
\vdots & \\
\boldsymbol{t}^{T}\left(\boldsymbol{P}^{N-1}\right)^{T} \boldsymbol{K}\left(\boldsymbol{P}^{N-1}\right) \boldsymbol{t} & =\frac{1}{\delta} .
\end{aligned}
$$

An approximate solution to (41) can be found by numerical optimization techniques, to be described next. First we define a sequence of matrices

$$
\boldsymbol{K}_{0}=\boldsymbol{K}, \boldsymbol{K}_{1}=\boldsymbol{P}^{T} \boldsymbol{K} \boldsymbol{P}, \cdots, \boldsymbol{K}_{N-1}=\left(\boldsymbol{P}^{N-1}\right)^{T} \boldsymbol{K}\left(\boldsymbol{P}^{N-1}\right)
$$

and $\boldsymbol{K}_{N}=\boldsymbol{K}_{0}$. Consider the following function of $\boldsymbol{t}$ :

$$
\phi(t)=\frac{\sum_{i=0}^{N-1}\left[\boldsymbol{t}^{T}\left(\boldsymbol{K}_{i}-\boldsymbol{K}_{i+1}\right) t\right]^{2}}{\sum_{i=0}^{N-1}\left[\boldsymbol{t}^{T}\left(\boldsymbol{K}_{i}\right) t\right]^{2}} .
$$

Note that $\phi(t) \geqslant 0$ for all nonzero $t$, and $\phi(t)=0$ if and only if

$$
\boldsymbol{t}^{T} \boldsymbol{K}_{0} \boldsymbol{t}=\boldsymbol{t}^{T} \boldsymbol{K}_{1} t=\cdots=\boldsymbol{t}^{T} \boldsymbol{K}_{N-1} \boldsymbol{t} .
$$

Equation (44) corresponds to the situation where (41) is satisfied with $1 / \delta$ set to $\boldsymbol{t}^{T} \boldsymbol{K}_{0} \boldsymbol{t}$. Therefore, the problem now is to find the global minimum of the function $\phi(t)$ with the constraint $t \neq 0$. Such a minimum will be the solution to (41).

An algorithm based on the method of steepest descent [22] is used for minimizing the function $\phi(t)$. For this method we need to know the gradient of $\phi(t)$, which can 
be computed from

$$
\begin{array}{r}
\left.\operatorname{grad}(\phi(t))\right|_{t=z_{0}}=\frac{4}{\beta}\left\{\sum_{i=0}^{N-1}\left(\alpha_{i}-\alpha_{i+1}\right)\left(\boldsymbol{K}_{i}-\boldsymbol{K}_{i+1}\right)\right. \\
\left.-\frac{\gamma}{\beta} \sum_{i=0}^{N-1} \alpha_{i} \boldsymbol{K}_{i}\right\} \boldsymbol{t}_{0}
\end{array}
$$

where

$$
\alpha_{i}=\boldsymbol{t}_{0}^{T} \boldsymbol{K}_{i} \boldsymbol{t}_{0}, \beta=\sum_{i=0}^{N-1} \alpha_{i}^{2} \text {, and } \gamma=\sum_{i=0}^{N-1}\left(\alpha_{i}-\alpha_{i+1}\right)^{2} \text {. }
$$

Let $t_{0}$ be an estimate of the minimum solution of $\phi(t)$.

1) At first a point is chosen at random to be $t_{0}$.

2) The direction of descent, denoted by $g$, is determined by the negative of the gradient evaluated at $t_{0}$, this can be computed using (45).

3) The step size, $s$, is set to a predetermined value.

4) Next we update $\boldsymbol{t}_{0}$ by replacing it with $\boldsymbol{t}_{0}+\boldsymbol{s g}$. If the improvement in the objective function is greater than a pre-specified amount $\epsilon$ then the next iteration is performed, otherwise the algorithm is terminated.

In the following example, we shall show the result of this method as applied to the circulant structure of (26b).

Example 4.1: Consider again the filter presented in Example 3.1. The circulant realization of this filter as given in (26b) is an unscaled realization since its $\boldsymbol{K}$-matrix is
Note that we can only manage to satisfy the scaling conditions of (37) approximately. However, for the simple case of a third-order filter it is possible to obtain an analytic solution to the problem of scaling. This is presented in Appendix A.

After scaling, the roundoff noise performance of the structure can be analyzed. There are different ways of placing quantizers within a filter, and here we choose to place them at the input of every delay element. This is the same as quantizing all the state variable nodes. Let $g_{i}(n)$ be the impulse response from the output of $i$ th quantizer to the overall filter output. Under standard assumptions [12], [13], the noise variance at the output can be computed as [13]

$$
\sigma_{0}^{2}=\sigma_{e}^{2} \sum_{i=1}^{N^{\prime}} \sum_{n=0}^{\infty} g_{i}^{2}(n)
$$

where $\sigma_{e}^{2}$ is the variance of the noise source. With roundoff arithmetic, we have $\sigma_{e}^{2}=2^{-2 b} / 12$ where $b$ is the number of binary bits used to represent internal signal precision.

As in [13], define the matrix $\boldsymbol{W}$ to be

$$
\boldsymbol{W}=\sum_{n=0}^{\infty} \boldsymbol{A}^{n^{T}} \boldsymbol{C}^{T} \boldsymbol{C A ^ { n }}
$$

$$
\boldsymbol{K}=2.6019\left(\begin{array}{rrrcc}
1.110 & 0.196 & -0.243 & 0.449 & 0.459 \\
0.196 & 0.937 & -0.231 & -0.192 & 0.410 \\
-0.243 & -0.231 & 0.929 & -0.485 & -0.411 \\
0.449 & -0.192 & -0.485 & 1.061 & 0.0869 \\
0.459 & 0.410 & -0.411 & 0.0869 & 0.926
\end{array}\right) .
$$

An approximate solution to (41) is obtained by the numerical optimization method outlined above, and the resulting circulant transformation is

$$
\boldsymbol{T}=\left(\begin{array}{ccccc}
-0.08466 & 0.1513 & 0.5779 & -0.1212 & 0.09243 \\
0.09243 & -0.08466 & 0.1513 & 0.5779 & -0.1212 \\
\vdots & \vdots & \vdots & \vdots & \vdots \\
0.1513 & 0.5779 & -0.1212 & 0.09243 & -0.08466
\end{array}\right) .
$$

By applying the transformation to (38), a scaled matrix $\boldsymbol{K}^{\prime}$ is obtained, and this is shown below:

$$
\boldsymbol{K}^{\prime}=\left(\begin{array}{cccrc}
1.000 & -0.509 & -0.432 & -0.164 & -0.259 \\
-0.509 & 1.000 & -0.0653 & 0.264 & -0.0899 \\
-0.432 & -0.0653 & 1.000 & 0.357 & 0.569 \\
-0.164 & 0.264 & 0.357 & 1.000 & 0.318 \\
-0.259 & -0.0899 & 0.569 & 0.318 & 1.000
\end{array}\right)
$$

The corresponding scaled structure is as follows:

$$
\begin{aligned}
\boldsymbol{A}^{\prime} & =\left(\begin{array}{ccccc}
0.8980 & -0.1905 & 0.0876 & -0.0854 & 0.1876 \\
0.1876 & 0.8980 & -0.1905 & 0.0876 & -0.0854 \\
\vdots & \vdots & \vdots & \vdots & \vdots \\
-0.1905 & 0.0876 & -0.0854 & 0.1876 & 0.8980
\end{array}\right) \\
\boldsymbol{B}^{\prime} & =\left(\begin{array}{lllll}
-0.20645 & 0.40645 & 0.33616 & 0.44859 & -0.00021368
\end{array}\right)^{T} \\
\boldsymbol{C}^{\prime} & =\left(\begin{array}{lllll}
0.22851 & 0.032972 & -0.1341 & 0.19058 & 0.20131
\end{array}\right) \\
d & =2.6019 \times 10^{-3} .
\end{aligned}
$$


One can verify that $[\boldsymbol{W}]_{i, i}=\sum_{n} g_{i}^{2}(n)$. Under the scaled condition of (37), the noise variance in (51) can now be expressed in terms of the diagonal elements of $\boldsymbol{W}$ and the diagonal elements of $\boldsymbol{K}$ :

$$
\boldsymbol{\sigma}_{0}^{2}=\boldsymbol{\sigma}_{e}^{2} \sum_{i=1}^{N}[\boldsymbol{W}]_{i, i}=\delta \boldsymbol{\sigma}_{e}^{2} \sum_{i=1}^{N}[\boldsymbol{K}]_{i, i}[\boldsymbol{W}]_{i, i} .
$$

Mullis and Roberts [13] have shown that for an arbitrary state-space realization the quantity in (53) has a lower bound, namely

$$
\sum_{i=1}^{N}[\boldsymbol{K}]_{i, i}[\boldsymbol{W}]_{i, i} \geqslant \frac{1}{N}\left(\sum_{i=1}^{N} \mu_{i}\right)^{2} .
$$

The $\mu$ 's are the eigenvalues of the matrix produce $\boldsymbol{K} \boldsymbol{W}$, and they are invariant under the similarity transformation of (4).

\section{Frequency Transformation on Circulant Structures}

An interesting invariant property arises for circulant and skew-circulant structures when one tries to perform frequency transformation on the filter transfer function $H(z)$. Let $\left\{\boldsymbol{A}_{0}, \boldsymbol{B}_{0}, \boldsymbol{C}_{0}, d_{0}\right\}$ be the state-space realization of $H(z)$. The above realization will have matrices $\boldsymbol{K}_{0}$ and $\boldsymbol{W}_{0}$ as defined in (36) and (52). Consider a new filter $G(z)$ which is derived from $H(z)$ by the transformation $G(z)=$ $H((\alpha-z) /(1-\alpha z))$, with $|\alpha|<1$. A state-space realization $\left\{\boldsymbol{A}_{1}, \boldsymbol{B}_{1}, \boldsymbol{C}_{1}, d_{1}\right\}$ for $G(z)$ can be constructed from [15]

$$
\begin{aligned}
& \boldsymbol{A}_{1}=\left(\alpha \boldsymbol{I}+\boldsymbol{A}_{0}\right)\left(\boldsymbol{I}+\alpha \boldsymbol{A}_{0}\right)^{-1} \\
& \boldsymbol{B}_{1}=\left(1-\alpha^{2}\right)^{(1 / 2)}\left(\boldsymbol{I}+\alpha \boldsymbol{A}_{0}\right)^{-1} \boldsymbol{B}_{0} \\
& \boldsymbol{C}_{1}=\left(1-\alpha^{2}\right)^{(1 / 2)} \boldsymbol{C}_{0}\left(\boldsymbol{I}+\alpha \boldsymbol{A}_{0}\right)^{-1} \\
& d_{1}=d_{0}-\alpha \boldsymbol{C}_{0}\left(\boldsymbol{I}+\alpha \boldsymbol{A}_{0}\right)^{-1} \boldsymbol{B}_{0} .
\end{aligned}
$$

Furthermore, it has been proved in [15] that $\boldsymbol{K}_{1}=\boldsymbol{K}_{0}$ and $\boldsymbol{W}_{1}=\boldsymbol{W}_{0}$. The sum or product of two circulant matrices is circulant, and the inverse of a circulant matrix, if it exists, is also circulant. Therefore, if $\boldsymbol{A}_{0}$ is a circulant matrix then $\boldsymbol{A}_{1}$ is circulant also. Similarly, if $\boldsymbol{A}_{0}$ is skew-circulant, then so is $\boldsymbol{A}_{1}$.

Now, if $\left\{\boldsymbol{A}_{0}, \boldsymbol{B}_{0}, \boldsymbol{C}_{0}, d_{0}\right\}$ represents a scaled circulant (skew-circulant) realization for $H(z)$, then $\left\{\boldsymbol{A}_{1}, \boldsymbol{B}_{1}, \boldsymbol{C}_{1}, d_{1}\right\}$ is a scaled circulant (skew-circulant) realization for $G(z)$, because we know that $\boldsymbol{K}_{1}=\boldsymbol{K}_{0}$. Furthermore, due to the fact $\boldsymbol{W}_{1}=\boldsymbol{W}_{0}$, both structures will have the same output noise variance as computed from (53). Hence we can conclude that the scaled circulant (skew-circulant) realizations of $H(z)$ and $G(z)$ have the same output noise variance irrespective of the value of $\alpha$.

\section{EXAMPles of Roundoff NoISE AND SENSITIVITY COMPARISONS}

Example 5.1: Consider the fifth-order low-pass elliptic filter presented in Example 3.1. The scaled circulant realization of this filter is shown in (50). The scaling is done

\begin{tabular}{|c|c|}
\hline Type of realization & $\begin{array}{c}\text { Noise } \\
\text { Variance } \\
\text { in } \mathrm{dB}\end{array}$ \\
\hline $\begin{array}{l}\text { Cascade of second order } \\
\text { canonic sections }\end{array}$ & -42.7 \\
\hline Circulant (unscaled) & -51.2 \\
\hline Circulant (scaied) & -51.7 \\
\hline Theoretical minimums & -52.1 \\
\hline
\end{tabular}
such that (37) holds with $\delta=1$. We shall compare the noise level in the scaled circulant form with the unscaled cir-
TABLE I

NOISE COMPARISON FOR FIFTH-ORDER ELLIPTIC FILTER

TABLE II

NOISE COMPARISON FOR SIXTH-ORDER BUTTERWORTH FILTER

\begin{tabular}{|c|c|}
\hline Type of realization & noise \\
variance \\
in dB
\end{tabular}

culant which is shown in (26). The unscaled structure is implemented by inserting a scale factor of $s$ at the input so as to reduce the maximum internal scaling norm to unity. This is compensated for by inserting a factor of $1 / s$ at the output, so that the overall signal level at the output remains unchanged. Throughout this example, the output signal level is the same for all structures under comparison. The same applies for Example 5.2 which follows. The output noise variance is calculated according to (51), with $\sigma_{e}^{2}=2^{-2 b} / 12$ and $b=7$. The result is listed in Table I for both the unscaled and scaled circulant forms. In order to provide a frame of reference, the noise variance of a cascaded structure of second-order canonic sections is also computed. The ordering of the second-order sections is chosen to be optimal. The minimum noise variance given 


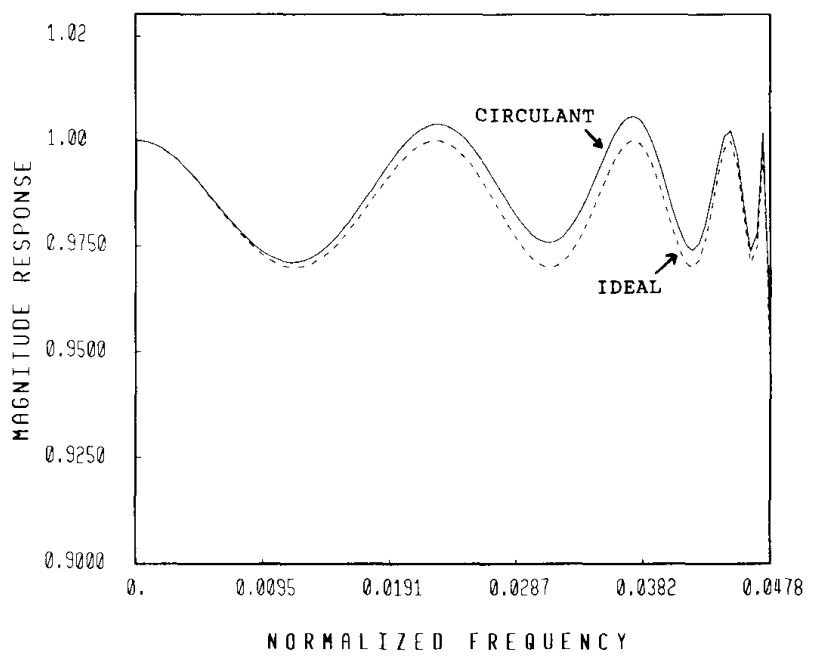

Fig. 1. Passband sensitivity of circulant form.

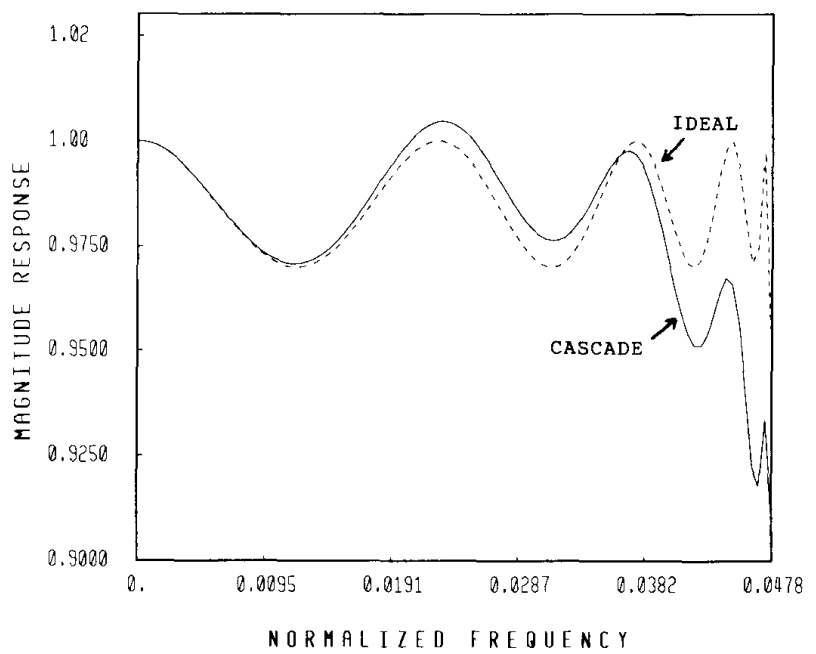

Fig. 2. Passband sensitivity of the cascade form.

by (54) for general state-space realization is included in Table I too. In this example, the scaled circulant structure gives us an improvement of $9 \mathrm{~dB}$ over the cascade, and it comes very close to achieving minimum noise.

Example 5.2: For the low-pass Butterworth filter presented in Example 3.2, (31b) shows the unscaled skew-circulant parameters of this filter. As in the previous example the structure was then scaled so that (37) holds with $\delta=1$. In Table II, we compare the output noise in several different structures: the skew-circulant form (both unscaled and scaled), a cascade of second-order canonic sections, and a cascade of second-order minimum-noise sections [3]. The ordering of the second-order sections is chosen to be optimal for each of the two cascade structures. Again the minimum noise variance, obtained from (54), is listed as reference. Table II shows that the scaled skew-circulant realization has a lower noise level than the cascade of canonic sections. However, it is not as good as the cascade of minimum-noise sections.

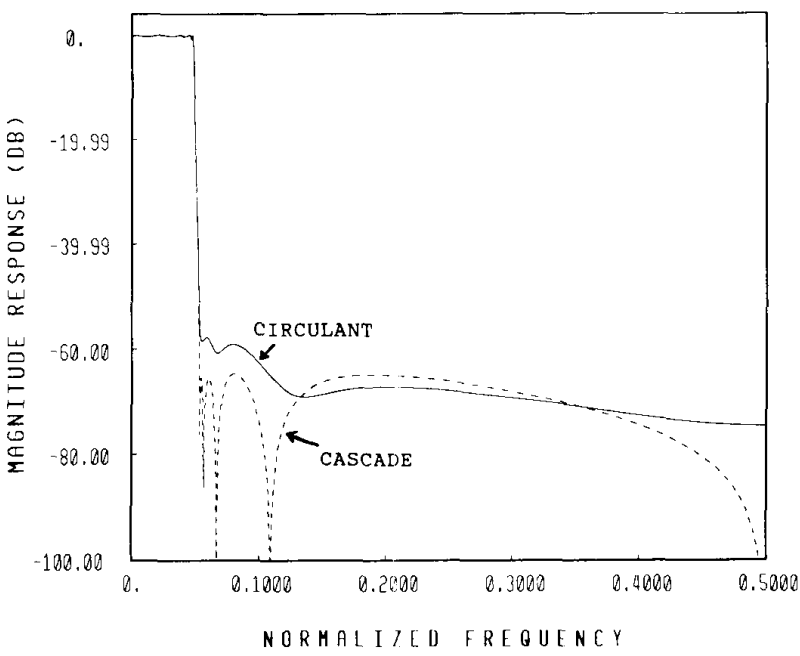

Fig. 3. Stopband comparison between cascade and circulant forms.

Example 5.3: With this example, we shall demonstrate the low passband sensitivity of the circulant form. The filter chosen is a ninth-order low-pass elliptic filter. The passband peak ripple and stopband attenuation are 0.265 and $60 \mathrm{~dB}$, respectively. The passband edge is at $0.096 \pi$, and the stopband starts at $0.106 \pi$. We implemented the filter using two different structures: one is a cascade of second-order canonic sections, while the other is the circulant state-space structure. After quantizing the multiplier coefficients to 5 bits in signed digit code, the passband response for both implementations are shown in Figs. 1 and 2. The ideal response is included in the graphs as reference. In the passband, the circulant filter has much better sensitivity than the cascade, while in the stopband (Fig. 3) the reverse is true.

\section{CONCLUSion}

This paper presents two new normal-form realizations of IIR digital filters. These are the circulant and skew-circulant form realizations, derived for the class of IIR filters for which the number of real poles does not exceed two. For other cases, we can obtain normal-form realizations which are parallel connections of smaller circulant sections. Example 5.3 demonstrates that the low eigenvalue sensitivity of these normal forms leads to low sensitivity in the passband magnitude response of the filter.

Scaling in these structures is accomplished by circulant or skew-circulant similarity transformations, since such transformations preserve the circulant or skew-circulant nature of the state transition matrix. Under scaled conditions, the output noise variances for the circulant and skew-circulant forms are shown to be invariant under low-pass-to-low-pass frequency transformation. This property can be useful for implementing narrow-band low-pass filters, since the noise variance here is insensitive to the bandwidth of the filter.

Examples show that in some cases the roundoff noise in the circulant and skew-circulant structures comes close to 
the minimum noise level derived in [13]. However, in other cases the noise of these structures is considerably higher than the minimum possible.

\section{APPENDIX A}

For the parallel-form implementation (21), its $\boldsymbol{K}$ matrix is given by

$$
[\boldsymbol{K}]_{i, j}=\frac{\beta_{i} \beta_{j}^{*}}{1-\lambda_{i} \lambda_{j}^{*}}
$$

Let $\left\{\boldsymbol{A}^{\prime}, \boldsymbol{B}^{\prime}, \boldsymbol{C}^{\prime}\right\}$ be a circulant structure derived from the parallel form via (22), then the matrix $\boldsymbol{K}^{\prime}$ is related to $\boldsymbol{K}$ as

$$
\boldsymbol{K}^{\prime}=\mathscr{W} \boldsymbol{K} \mathscr{W}^{\dagger} .
$$

Our goal is to make all the diagonal elements of $\boldsymbol{K}^{\prime}$ equal to a constant $1 / \delta$, so that the realization $\left\{\boldsymbol{A}^{\prime}, \boldsymbol{B}^{\prime}, \boldsymbol{C}^{\prime}\right\}$ is scaled according to (37). This can be accomplished by choosing the parallel-form parameters (21), $\beta_{i}$ and $\gamma_{i}$, appropriately as shown next. From (A2)

$$
\left[\boldsymbol{K}^{\prime}\right]_{i, i}=\sum_{j=0}^{N-1} \sum_{k=0}^{N-1} W^{-i(k-j)}[\boldsymbol{K}]_{j, k} .
$$

With the change of variables, $m=k-j$,

$$
\begin{aligned}
{\left[\boldsymbol{K}^{\prime}\right]_{i, i} } & =\sum_{j=0}^{N-1} \sum_{m=-j}^{N-1-j} W^{-i m}[\boldsymbol{K}]_{j, j+m} \\
& =\sum_{j=0}^{N-1} \sum_{m=0}^{N-1} W^{-i m}[\boldsymbol{K}]_{j,((j+m))} .
\end{aligned}
$$

The notation $((j+m))$ stands for $j+m$ modulo $N$. Define a $N$ point sequence

$$
v(m)=\sum_{j=0}^{N-1}[\boldsymbol{K}]_{j,((j+m))}
$$

then

$$
\left[\boldsymbol{K}^{\prime}\right]_{i, i}=\sum_{m=0}^{N-1} W^{-i m} v(m) .
$$

The diagonal elements of $\boldsymbol{K}^{\prime}$ are related to $v(m)$ by the inverse DFT. Since condition (37) requires $\left[\boldsymbol{K}^{\prime}\right]_{i, i}$ be the same for all $i$, we should have $v(0)=1 / \delta$ and $v(m)=0$ for $m=1, \cdots, N-1$. In terms of the $\beta$ 's and $\lambda$ 's in (21), this means

$$
\sum_{j=0}^{N-1} \frac{\beta_{j} \beta_{((j+m))}^{*}}{1-\lambda_{j} \lambda_{((j+m))}^{*}}= \begin{cases}\frac{1}{\delta}, & \text { for } m=0 \\ 0, & \text { otherwise. }\end{cases}
$$

The values of $\lambda$ 's are fixed, so the only free variables are the $\beta$ 's. This leaves us with $N$ equations and $N$ unknowns.

For the simple case of a third-order filter $(N=3)$, a closed-form solution for (A7) can be found. Assuming $\lambda_{0}, \beta_{0}$ are real, $\lambda_{1}=\lambda_{2}^{*}$, and $\beta_{1}=\beta_{2}^{*}$, (A7) is reduced to

$$
\begin{aligned}
& \frac{\beta_{0}^{2}}{1-\lambda_{0}^{2}}+\frac{2\left|\beta_{1}\right|^{2}}{1-\left|\lambda_{1}\right|^{2}}=\frac{1}{\delta} \\
& \frac{2 \beta_{0} \beta_{1}^{*}}{1-\lambda_{0} \lambda_{1}^{*}}+\frac{\beta_{1}^{2}}{1-\lambda_{1}^{2}}=0 \\
& \frac{2 \beta_{0} \beta_{2}^{*}}{1-\lambda_{0} \lambda_{2}^{*}}+\frac{\beta_{2}^{2}}{1-\lambda_{2}^{2}}=0 .
\end{aligned}
$$

Equation (A8c) is redundant, so we only need to solve (A8a) and (A8b). The result is

$$
\begin{aligned}
\beta_{0} & = \pm \frac{1}{\delta}\left[\frac{8}{1-\left|\lambda_{1}\right|^{2}} \cdot\left|\frac{\lambda_{1}^{2}-1}{1-\lambda_{0} \lambda_{1}^{*}}\right|^{2}+\frac{1}{1-\lambda_{0}^{2}}\right]^{-(1 / 2)} \\
\left|\beta_{1}\right| & =2\left|\frac{\lambda_{1}^{2}-1}{1-\lambda_{0} \lambda_{1}^{*}}\right| \cdot\left|\beta_{0}\right| \\
\arg \left(\beta_{1}\right) & =\left\{\begin{array}{l}
\frac{1}{3}\left[\arg \left(\frac{\lambda_{1}^{2}-1}{1-\lambda_{0} \lambda_{1}^{*}}\right)+\arg \left(\beta_{0}\right)\right] \\
\left.\frac{1}{3}\left[\arg \left(\frac{\lambda_{1}^{2}-1}{1-\lambda_{0} \lambda_{1}^{*}}\right)+\arg \left(\beta_{0}\right)+2 \pi\right] . \quad \text { (A9 }\right) \\
\frac{1}{3}\left[\arg \left(\frac{\lambda_{1}^{2}-1}{1-\lambda_{0} \lambda_{1}^{*}}\right)+\arg \left(\beta_{0}\right)+4 \pi\right]
\end{array}\right.
\end{aligned}
$$

Altogether there are six solutions to (A8). In summary, for a third-order filter if $\beta_{0}, \beta_{1}$ and $\beta_{2}$ are chosen according to (A9), then the circulant form that is derived from (21) will automatically be scaled.

\section{REFERENCES}

[1] C. W. Barnes and A. T. Fam, "Minimum norm recursive digital filters that are free of overflow limit cycles," IEEE Trans. Circuits Syst., vol. CAS-24, pp. 569-574, Oct. 1977 .

[2] C. W. Barnes, "A parametric approach to the realization of second-order digital filter sections," vol. CAS-32, pp. 530-539, June 1985.

[3] W. L. Mills, C. T. Mullis, and R. A. Roberts, "Low roundoff noise and normal realization of fixed point IIR digital filters," IEEE Trans. Acoust., Speech, Signal Processing, vol. ASSP-29, pp. 893-903, Aug. 1981

[4] C. M. Rader and B. Gold, "Effects of parameter quantization on the poles of a digital filter," Proc IEEE, vol. 55, pp. 688-689, May 1967.

[5] A. Antoniou, Digital Filters: Analysis and Design. New York: McGraw-Hill, 1979, pp. 22-26.

[6] L. B. Jackson, "Limit cycles in state-space structures for digital filters," IEEE Trans. Circuits Syst., vol. CAS-26, pp. 67-68, Jan. 1979.

[7] W. L. Mills, C. T. Mullis and R. A. Roberts, "Digital filter realizations without overflow oscillation," IEEE Trans. Acoust., Speech, Signal Processing, vol. ASSP-26, pp. 334-338, Aug. 1978.

[8] J. N. Franklin, Matrix Theory. Englewood Cliffs, NJ: PrenticeHall, 1968, pp. 115-120.

[9] A. S. Deif, Advanced Matrix Theory for Scientists and Engineers. Tunbridge Wells, England: Abacus, 1982, pp. 230-232; NJ: Van Nostrand, 1958.

[10] R. E. Blahut, Fast Algorithms for Digital Signal Processing. New York, Addison-Wesley, 1985, pp. 65-113.

[11] A. Peled and B. Liu, "A new hardware realization of digital filters," IEEE Trans. Acoust., Speech, Signal Processing, vol. ASSP-22, pp. 456-462, Dec. 1974. 
[12] L. B. Jackson, "On the interaction of roundoff noise and dynamic range in digital filters," Bell Syst. Tech. J., vol. 49, pp. 159-184, 1970 .

[13] C. T. Mullis and R. A. Roberts, "Synthesis of minimum roundoff noise fixed point digital filters," IEEE Trans. Circuits Syst., vol. CAS-23, pp. 551-562, Sept. 1976.

[14] P. J. Davis, Circulant Matrices. New York, Wiley, 1979

[15] C. T. Mullis and R. A. Roberts, "Roundoff noise in digital filters: Frequency transformations and invariants," IEEE Trans. A coust. Speech, Signal Processing, vol. ASSP-24, pp. 538-550, Dec. 1976.

[16] P. P. Vaidyanathan and V. C. Liu, "An improved sufficient-condition for absence of limit-cycles in digital filters," IEEE Trans. Circuits Syst., vol. CAS-34, pp. 319-322, Mar. 1987.

[17] A. V. Oppenheim and R. W. Schafer, Digital Signal Processing. Englewood Cliffs, NJ: 1975

[18] B. Gold and K. L. Jordan, "A note on digital filter synthesis," Proc. IEEE, vol. 65, pp. 1717-1718, Oct. 1968.

[19] C. S. Burrus "Block implementation of digital filters" IEEE Trans. Circuits Theory, vol. CT-18, pp. 697-701, Nov. 1971.

[20] S. K. Mitra and R. Gnanasekaran, "Block implementation of recursive digital filters- New structures and properties," IEEE Truns. Circuits Syst., vol. CAS-25, pp. 200-207, Apr. 1978

[21] C. W. Barnes, "Finite word effects in block-state realizations of fixed-point digital filters," IEEE Trans. Circuits Syst., vol. CAS-27, pp. 345-349, May 1980

[22] P. Gill, W. Murray, and M. Wright, Practical Optimization. New York: 1981 .

隶

Vincent C. Liu (S'85) was born in Hong Kong on November 15, 1961. He received the B.E. degree in electrical engineering from the State University of New York at Stony Brook in 1984, and the M.S. degree from the California Institute of Technology, Pasadena, in 1985. He is currently a



Ph.D. candidate at the California Institute of Technology. His main research interests are in the areas of digital signal processing, non-linear effects in digital filters and two-dimensic ral lin ear discrete-time systems.

Mr. Liu is the recipient of a Schlumberger Fellowship, and is a member of Tau Beta $\mathrm{Pi}$.



P. P. Vaidyanathan (S'80-M'83-SM'88) was born in Calcutta, India, in 1954 . He received the B.Sc. (hon) degree in physics, the B. Tech. and $M$

Tech. degrees in radiophysics and electronics from the University of Calcutta, India, in 1974 1977, and 1979, respectively, and the Ph.D. degree in electrical and computer engineering from the University of California, Santa Barbara, in 1982.

He was a Post-Doctoral Fellow at the University of California, Santa Barbara, from September 1982 to February 1983. Since March 1983 he has been with the California Institute of Technology, Pasadena, as an Assistant Professor of Electrical Engineering. His main research interests are in digital signal processing, linear systems, and filter design.

Dr. Vaidyanathan served as the Vice-Chairman of the Technical Program Committee for the 1983 IEEE International Symposium on Circuits and Systems. He currently serves as an Associate Editor for the IEEE Transactions on Circuits and Systems. He was the recipient of the Award for Excellence in Teaching at the California Institute of Technology for the years 1983/1984. He was also a recipient of the NSF's Presidential Young Investigator A.ward, starting from the year 1986. 